\title{
ENFERMEDAD DE VON GIERKE: NUEVAS TENDENCIAS EN EL MANEJO
}

\author{
Jorge Armando Daza Cárdenas \\ Médico y Cirujano \\ Universidad Militar Nueva Granada \\ Recibido: Febrero 13 de 2012 Aceptado: Mayo 23 de 2012
}

\begin{abstract}
Resumen
La enfermedad de von Gierke, también conocida como enfermedad de deposito de glucógeno tipo la, es una enfermedad producida por la deficiencia de la unidad catalítica de la G6Pasa-a, encargada de hidrolizar la glucosa 6 fosfato en el citoplasma celular durante la gluconeogénesis y la glucogenolisis. Las complicaciones a largo plazo son hipoglicemia severa y alteraciones en el crecimiento. En los niños más pequeños la enfermedad típicamente se presenta con crisis convulsivas y hepatomegalia que se manifiestan a los 6 y 8 meses. Otras complicaciones son osteoporosis, gota, enfermedad renal, hipertensión pulmonar y adenomas hepáticos que pueden malignizarse. No se ha encontrado una cura y de no recibir un manejo adecuado es letal en las primeras dos décadas de la vida. El tratamiento consiste en terapia nutricional, asociada a varios medicamentos convencionales. Algunos pacientes pueden requerir transplante renal o transplante hepático. Una nueva esperanza se ha abierto con el advenimiento de la terapia génica con vectores virales, esta estrategia hasta ahora esta siendo desarrollada, pero los estudios realizados han mostrado una luz de esperanza para investigadores, médicos y pacientes. Faltan estudios para que estos tratamientos permitan un beneficio a largo plazo y su aplicación en humanos, ya que las pruebas como es de esperarse solo han sido desarrolladas en modelos animales.
\end{abstract}

Palabras clave: Deficiencia glucosa 6 fosfato, enfermedad de von Gierke, enfermedad de depósito de glicógeno tipo I, terapia transgénica, carcinoma hepatocelular.

\section{VON GIERKE DISEASE: NEW TRENDS IN MANAGEMENT}

\begin{abstract}
Von Gierke disease, also known as glycogen storage disease type la, is a disease caused by deficiency of the G6Pase-a catalytic unit, which hydrolyzes glucose-6- phosphate in the cell cytoplasm during gluconeogenesis and glycogenolysis. Long term complications include severe hypoglycemia and growth disturbances. In small children, the disease typically presents with seizure crisis and hepatomegaly which become manifest at the age of 6 and 8 months. Other complications include osteoporosis, gout, renal disease, pulmonary hypertension and hepatic adenomas which can become malignant. No cure has been found for this disease and it can turn out to be lethal if no appropriate management is given during the first two decades of life. The treatment consists of nutritional therapy associated with a number of conventional drugs. Some patients may require renal or liver transplant. A new hope has emerged with the arrival of gene therapy with viral vectors, strategy that is being developed hitherto, yet performed studies have shown a glimmer of hope for investigators, doctors and patients. There is a need for studies so these treatments allow for a longer term benefit and their application in humans since, as expected, the tests have been developed only in animal models.
\end{abstract}

Key words: Glucose-6-phosphatase deficiency, von Gierke disease, glycogen storage disease type I (GSD I), transgenic therapy, hepatocellular carcinoma.

* Correspondencia: jorgedaza87@gmail.com 


\title{
DOENÇA DE VON GIERKE: NOVAS TENDÊNCIAS NO TRATAMENTO
}

\begin{abstract}
RESUMO
A doença de Von Gierke, também conhecida como Glicogenose tipo I, é uma doença produzida pela deficiência da unidade catalítica da G6Pasa-a, encarregada de hidrolisar a glicose 6 fosfato no citoplasma celular durante a gliconeogênese e a glicogenólise. As complicações a longo prazo são hipoglicemia severa e alterações no crescimento. Nas crianças menores a doença se apresenta tipicamente com crises convulsivas e hepatomegalia que se manifestam aos 6 e 8 meses. Outras complicações são osteoporose, gota, doença renal, hipertensão pulmonar e adenomas hepáticos que podem malignizar-se. Não foi encontrada uma cura e se não recebe tratamento adequado é letal nas primeiras duas décadas de vida. O tratamento consiste em terapia nutricional, associada a vários medicamentos convencionais. Alguns pacientes podem requerer transplante renal ou transplante hepático. Uma nova esperança apareceu com a terapia gênica com vetores virais, esta estratégia até agora esta sendo desenvolvida, mas os estudos realizados mostram uma luz de esperança para pesquisadores, médicos e pacientes. Faltam estudos para que estes tratamentos permitam um beneficio a longo prazo e a sua aplicação em humanos, já que os testes como é de se esperar só foram desenvolvidos em modelos animais.
\end{abstract}

Palavras chave: Deficiência glicose 6 fosfato, doença de von Gierke, glicogenose I, terapia transgênica, carcinoma hepatocelular.

\section{Introducción}

Las enfermedades de depósito del glucógeno tipo I son un grupo de trastornos autosómicos recesivos causados por deficiencia del complejo glucosa-6-fosfatasa-alfa (G6Pasa-a). Dos enzimas están implicadas en esta alteración,el transportador de glucosa-6-fosfato (G6PT) que transtoca este sustrato desde el citoplasma al lumen del retículo endoplamico y la unidad catalítica de la G6Pasa-a que hidroliza la G6P y constituye el paso final de la gluconeogénesis y glucogenólisis (1). Debido a lo anterior el daño de la enzima conllevará a un acumulo de G6P en el citoplasma activando vías metabólicas secundarias que la usan como sustrato. La homeostasis de la glicemia entre cada comida es responsabilidad del G6Pasa-a. La deficiencia de la unidad catalítica de la G6Pasa-a causa la glucogenosis tipo Ia y la deficiencia en la G6PT causa la glucogenosis tipo Ib (2).

\section{Genética}

La G6Pasa-a se expresa predominantemente en el hígado, los riñónes y en elintestino. Por su parte la G6PT se expresa en todos los tejidos. Ambas alteraciones no permiten la hidrólisis de la G6P y los pacientes adquieren un fenotipo especial por el trastorno de la homeostasis glucémica (1). Los genes que codifican la G6Pasa y del G6PT se encuentran en los cromosomas $17 q 21$ y 11q23 respectivamente (3). Se han identificado mas de 85 mutaciones la mayoría son mutaciones tipo missense, aunque también se han evidenciado otro tipo de mutaciones tipo Nonsense y frameshit.

\section{Epidemiología}

Las enfermedades de deposito tipo I tienen una incidencia estimada de 1 en 100000 a 1 en 200000 nacidos vivos a nivel mundial. La incidencia mas alta se observa en los judíos Ashkenazi con una frecuencia de portador de 1:65 lo cual permite predecir una prevalencia cinco veces mayor que en la población caucásica en general (4). De los subtipos ya mencionados la glucogenosis tipo la es la más prevalente y representa el $80 \%$ de los casos (1).

\section{Manifestaciones clínicas}

Tanto la glucogenosisla y Ib se caracterizan por hipoglicemia con ayuno no muy prolongado. El uso de vías metabólicas alternativas producen hipertrigliceridemia, hipercolesterolemia, hiperuricemia y acidemialactica (5). A diferencia de la glucogenosis tipo Ia, la tipo Ib se acompaña de neutropenia y disfunciones de la línea mieloide, que no se relacionan con el metabolismo de los órganos gluconeogénicos (1).

Los pacientes presentan retraso del crecimiento, obesidad centrípeta, hemorragias por disfunción plaquetaria, hepatomegalia y aumento del tamaño renal por exceso 
en el depósito de glucógeno en estos órganos (6). Este último a su vez produce acidosis tubular renal con perdida de bicarbonato de sodio y un elevado nivel de acido úrico en orina. Adicionalmente se encuentran niveles elevados de lactato, originando una acidosis con anion gap elevado. Frecuentemente estos pacientes presentan irritabilidad, hipotonía e hipo ventilación secundarios a la hipoglicemia (7).

\section{Diagnóstico}

El diagnóstico se hace mediante el hallazgo de hipoglicemia, hiperlactatemia, hipertrigliceridemia, hipercolesterolemia y en muchos casos hiperuricemia. Adicionalmente, se realizan test funcionales que demuestran la ausencia de una respuesta hiperglicémica en pacientes a quienes se inyecta $1 \mathrm{mg} / \mathrm{m} 2$ de glucagón en ayunas o después de 2 horas de haber ingerido alimentos ricos en carbohidratos. La presencia de hipoinsulinemia, hipoacetonemia e hiperglucagonemia así como un aumento en la actividad de la biotinidasa sérica, también ayudan a orientar el diagnóstico (8).

El diagnóstico bioquímico de la enfermedad, requiere una biopsia hepática idealmente no congelada, donde se mide la actividad hidrolítica usando manosa 6 fosfato, G6P y pirofosfato. En la glucogenosis tipo Ia se evidencia una defectuosa actividad hidrolítica, sin importar el sustrato utilizado y el estado de la membrana microsomal, a diferencia de la glucogenosis tipo $\mathrm{Ib}$ donde se ve una alteración de la actividad hidrolítica con membranas microsomales intactas. Además, en el estudio histopatológico se evidencia distención hepática inducida, por acúmulo de lípidos y glucógeno y en algunos casos puede presentarse fibrosis (8).(VIII)

\section{Complicaciones}

Las complicaciones a largo plazo son hipoglicemia severa, alteraciones en el crecimiento y crisis convulsivas que se manifiestan a los 6 y 8 meses. Otras complicaciones son osteoporosis, anemia refractaria al tratamiento con hierro, hiperuricemia, falla renal, hipertensión pulmonar y adenomas hepáticos que pueden malignizarse (9). Se recomienda en los primeros diez años de vida realizar una ecografía hepatobiliar anual y después de estos primeros años, una resonancia magnética anual. Si en alguno de estos controles se hallan lesiones hepáticas, deben realizarse controles cada 6 meses. Estas manifestaciones aunque inevitables, pueden hacer una aparición mas lenta, si el paciente tiene un adecuado control metabólico (8).

\section{Tratamiento}

Para ninguna de estas dos enfermedades hay cura $y$ de no ser tratadas son fatales en las primeras dos décadas de la vida. El tratamiento consiste en manejo nutricional, asociado a varios fármacos convencionales. Los pacientes que por algún motivo no pueden ingerir alimentos, reciben una infusión de glucosa por sonda nasogástrica o por vía parenteral, y los pacientes que toleran vía oral reciben maicena no cocinada (1.6-2.5g/ $\mathrm{kg}$ ), inicialmente cada 3-4 horas y eventualmente cada 6 horas. La maicena actúa como un carbohidrato de liberación lenta, y permite prolongar el tiempo de euglicemia entre las comidas. Este manejo permite a los pacientes alcanzar un crecimiento y desarrollo puberal casi normal. Sin embargo, la enfermedad no se corrige y los paciente continúan presentando, hiperlipidemia, hipercalciuria, hiperuricemia, hipocitraturia y acidemialáctica. La dieta no previene todas las complicaciones a largo plazo (10). El control de la ingesta calórica debe ser monitoreado rigurosamente, ya que una ingesta insuficiente no corrige el desorden metabólico y lleva a un retardo del crecimiento, y una ingesta excesiva causa obesidad. La dieta debe distribuirse de la siguiente forma: 60 - 65\% de carbohidratos, 10 - 15\% de proteínas y el restante lípidos (8).

Sin embargo, este tratamiento nutricional resulta muy dispendioso y puede llegar a alterar de manera sustancial la calidad de vida de los pacientes y sus acudientes, debido a que la maicena no procesada debe ser administrada múltiples veces a lo largo del día. Teniendo en cuenta ésto, resulta necesario iniciar la investigación de nuevas sustancias que permitan disminuir el número de dosis necesarias a lo largo del día. Un estudio de Correia y colaboradores realizado 2008, en el cual se analizó el uso de maicena procesada con calor húmedo, evidencio una reducción significativa en el numero de episodios de hipoglicemia en la noche. También se encontró que esta sustancia previene con más efectividad, las crisis de hipoglicemia a lo largo del día, y que el incremento en las cifras de glicemia es mas lento así como su disminución. Los pacientes que fueron tratados con este tipo de maicena y que disminuían sus niveles de glicemia por debajo de 70 $\mathrm{mg} / \mathrm{dl}$, disminuían aún más la velocidad de descenso, demostrando así, que el olvido de alguna dosis no seria tan letal como lo es en la actualidad. Los beneficios de esta sustancia experimental en conclusión serian una mayor seguridad y una mejor calidad de vida (11). El seguimiento debe hacerse mediante la evaluación de 
la curva de crecimiento, el índice de masa corporal, la medición de la hepatomegalia y la presión arterial. La glicemia debe mantenerse por encima de $3.5 \mathrm{mmol} / \mathrm{L}$. Los niveles de lactato, de ser posible, también deben ser monitorizados, así como debe realizarse un cuadro hemático, un perfil lipídico, uricemia, gases sanguíneos y proteinuria en cada consulta (8).

Los pacientes que presentan microalbuminuria deben iniciar tratamiento inmediato con inhibidores de la enzima convertidora de angiotensina (IECA), así como debe analizarse si es necesario el uso de alopurinol, hierro, calcio y suplementos vitamínicos según el estado bioquímico y metabólico del paciente. El uso de fármacos reductores de niveles de triglicéridos, no esta indicado si los niveles permanecen por debajo de $10 \mathrm{mmol} / \mathrm{L}$. Los fármacos reductores de colesterol no están indicados en pacientes jóvenes, por que tienen bajo riesgo de aterogenicidad (8).

Con el incremento en la sobrevida de estos pacientes se ha visto un aumento en la incidencia de carcinoma hepatocelular, y que en la mayoría de estos pacientes se presentaban adenomas hepáticos previos. El promedio de edad para la aparición del cáncer es de 28 años (6). Algunos pacientes pueden requerir transplante renal o hepático. El transplante hepático parece corregir las alteraciones metabólicas a corto plazo, pero no hay estudios que demuestren lo mismo a largo plazo. Aún así, el riesgo para la vida del receptor de un transplante de órgano es muy alto, por lo que se requieren otras opciones de tratamiento. Los pacientes llevados a estos procedimientos deben tener un control estricto de los niveles de glucosa y lactato (6).

El pronóstico de estos pacientes mejora notoriamente con el inicio temprano de una adecuada terapia nutricional, y muchos de ellos pueden llevar una vida casi normal. Si se mantiene la normoglicemia, las anormalidades metabólicas y los parámetros clínicos mejoran, aunque la dislipidemia no se corrige. La incidencia de adenomas hepáticos parece disminuir, aunque la progresión de la enfermedad renal no se detiene. Algunos pacientes a pesar de un adecuado manejo, continúan con retraso en el crecimiento y pueden requerir transplante hepático y renal. En los pacientes con glucogenosis tipo Ib la normalidad es más difícil de obtener y de mantenerse por lagos periodo de tiempo, por la predisposición de estos pacientes a infecciones severas (8).

\section{Terapia génica}

Mediante estudios realizados en ratones y perros se ha logrado demostrar, que se necesita un $7 \%$ de la actividad hepática normal para corregir la glucogenosis tipo Ia. En el 2000 Zingone et al. evaluaron la posibilidad de realizar un reemplazo génico como terapia para esta patología, usando como transportador de G6Pasa-a un vector adenovírico. Una infusión simple de este tratamiento restauro 19\% de la actividad G6Pasa-a del hígado, mejoro la supervivencia y el crecimiento en estos ratones y corrigió transitoriamente las anormalidades metabólicas que se habían desarrollado. Sin embargo, todos estos beneficios se presentaban en un periodo de tiempo muy corto debido a la rápida eliminación del gen transportado en el vector (1).

Estudios realizados en perros, a los cuales se les infundio G6Pasa-a usando un virus adeno-asociado tipo 2, demostraron que la supervivencia no depende de la dosis infundida del medicamento así como tampoco del grado de restauración de la función G6Pasa-a hepática. Se encontró, que la normalización del 10.65 de la función hepática, es suficiente para normalizar los valores de glicemia en ayunas. No se logró un adecuado control de la cifras de colesterol, triglicéridos y lactato con el tratamiento (1).

Por otra parte, un estudio realizado por Koeberl y colaboradores en el 2008 demostró que la introducción de un vector que codifica la G6Pasa en modelos murinos, aumentó la supervivencia con dosis aceptables para uso clínico. El promotor humano usado para la expresión, actuó suficientemente regulado como para permitir la corrección de la hipoglicemia, sin causar hiperglicemia. Así, la deficiencia de la enzima fue completamente restablecida, mientras que en estudios previos solo se logro obtener del 10 al $20 \%$ de la función normal en modelos experimentales. Así mismo, se evidencio una expresión transgénica sostenida con mínimo infiltrado linfocitario, lo que demuestra una adecuada tolerancia del sistema inmune de los ratones y caninos. En cuanto a la estabilidad, se vio que la expresión se mantenía por 52 semanas en la mayoría de los modelos, pero en algunos, se vio una actividad estable permanentemente. Se observó, que la estabilización de los niveles de glicemia permite corregir adecuadamente los otros trastornos metabólicos observados en esta enfermedad, como ocurre en pacientes con trasplante hepático. El retraso en el crecimiento puede ser corregido, siempre y cuando, la terapia génica se inicie a tempranamente en los ratones y caninos (12). 
Se ha estudiado múltiples modelos que permitan mejorar la reproducibilidad de los resultados obtenidos en los estudios, el modelo canino de glucogenosis la tiene un componente genético y metabólico similar al de los humanos, también se ha observado similitudes en las manifestaciones clínicas y patológicas halladas en caninos, mucho mayores que las evidenciadas en murinos. Otras ventajas que ofrecen los perros son su expectativa de vida mas larga y un mayor tamaño, que permite observar con mayor claridad los resultados. Las mayores desventajas que se observaron en este tipo de huéspedes son que requieren mayor tiempo y costo en su cuidado (13).

\section{Diagnóstico diferencial}

Generalmente estas enfermedades analizadas son muy claras en la infancia, y no es difícil llegar al diagnóstico. Sin embargo, en algunos pacientes mayores puede complicarse el diagnóstico si tienen mayor tolerancia al ayuno. La deficiencia de fructosa 1,6 difosfatasa puede producir algunos de los signos y síntomas de las enfermedades de deposito del glucógeno tipo la y $\mathrm{Ib}$, sin embargo, los pacientes que la padecen tienen mayor tolerancia al ayuno.

Por otro lado, la glucogenosis tipo III clínicamente también puede presentarse como la enfermedad de von Gierke, pero la hipoglicemia no es tan severa por que la gluconeogénesis no esta afectada, la lactatemia en ayunas y el acido úrico son normales generalmente pero las transaminasas esta elevadas.

Tumores hepáticos primarios así como metastásicos son fácilmente descartables por la presentación clínica y la ultrasonografía (8)

\section{Conclusiones}

Desde la descripción del primer caso de glucogenosis tipo I, el manejo ha evolucionado a pasos agigantados. Los avances en el tratamiento, como es de esperarse, se han visto reflejados en un mejor pronostico para los pacientes. Además, de contar con cada vez mejores tratamientos nutricionales para mantener la euglicemia de los pacientes, se siguen realizando estudios para obtener terapias alternativas en este campo, que afecten en menor medida su calidad de vida y mejore su adherencia al tratamiento. Con el pasar de los últimos años, se ha despertado más el interés de la comunidad científica por encontrar terapias menos invasivas, con mayor estabilidad, con mejor pronóstico, no solo en el corto sino también en el largo plazo. La terapia transgénica ha sido ampliamente estudiada en los últimos años, aunque faltan mas trabajos para empezar a aplicarlos y evaluar, obviamente, su efectividad en humanos. Sin embargo, observando cronológicamente la literatura científica, se observan cada vez mas avances en cuanto a estabilidad de vectores y resultados en modelos bastantes similares al modelo humano, como es el caso de los murinos y caninos.

\section{Referencias}

1. Chou J, Mansfield B. Gene therapy for type I glycogen storage diseases. Curr Gene Ther. 2007 Abr; 7(2): 79-88.

2. Chou J, Mansfield B. Mutations in the glucose- 6 phosphatase- $\square$ (G6PC) gene that cause type la glycogen storage disease. Hum Mutat. 2008 Jul; 29(7): 921-930.

3. Heller S, Worona L, Consuelo A. Nutritional therapy for glycogen storage. Diseases Journal of Pediatric Gastroenterology and Nutrition. 47: S15-S21.

4. Weinstein D, Wolfsdorf J. Glycogen storage diseases: A primer for clinicians. The Endocrinologist. 2002; 12: 531-538.

5. Wierzbicki AS, Watt GF, Lynas J, Winder AF, Wray R. Very lowdensity lipoprotein apolipoprotein B-100 turnover in glycogen storage disease type Ia (von Gierke disease). J Inherit Metab Dis. 2001 Oct; 24(5): 527-34.

6. Oshita A, ItamotoT, AmanoH, Ohdan H, TashiroH, AsaharaT Perioperative management of benign hepatic tumors in patients with glycogen storage disease type Ia. J Hepatobiliary Pancreat Surg. 2008; 15: 200-203.

7. Hinrichs J, Dever E. History matters: A 4-Month-Old infant with a diagnosis of von Gierke's disease. J Emerg Nurs. 2006; 32: 343-6.

8. Froissar R, Piraud M, Mollet A, et al. Glucose-6-phosphatase deficiency. Orphanet Journal of Rare Diseases. 2011; 6: 27.

9. Albert G, Reba C, Simon N. Waddington neonatal gene therapy of glycogen storage disease type la using a feline immunodeficiency virus-based vector. Molecular Therapy. 2010; 18(9):

10. Koeberl, D, KishnaniP, Chen Y. Glycogen storage disease types I and II: Treatment updates. J Inherit Metab Dis. 2007 Abr; 30(2): 159-164.

11. Correia C, Bhattacharya K, Lee P, ShusterJ, Theriaque D, et al. Of modified cornstarch therapy to extend fasting in glycogen storage disease types Ia and IbAm. J Clin Nut. 2008; 88:1272-6.

12. Dwight D, Koeberl 1, Carlos Pinto 2, Baodong S, et al. AAV vector-mediated reversal of hypoglycemia in canine and murine glycogen storage disease type Ia. Molecular Therapy. 2008 abr.;16(4): 665-672.

13. Specht A,1 Fiske L,2 Erger K. Glycogen storage disease type Ia in canines: A model for human metabolic and genetic liver disease. J Biomed Biotechnol. 2011; 2011: 646257. Epub 2011. 